\title{
Variación del módulo de Young con el tratamiento térmico en aceros al carbono hipoeutectoides
}

\author{
A. Villuendas*, J. Jorba** y A. Roca* \\ Resumen En trabajos anteriores se ha constatado que varios aceros al carbono hipoeutectoides, en \\ estado de temple, presentan valores del módulo de Young inferiores a los correspondientes \\ en estado de revenido. En todos los casos la determinación se ha realizado mediante \\ ultrasonidos. En concreto, para el acero C22E (EN 10083), el módulo se incrementa \\ ligeramente desde $209 \mathrm{GPa}$ (material templado) hasta $211 \mathrm{GPa}$ (revenido a $650^{\circ} \mathrm{C}$ ), para \\ el acero C45E el módulo aumenta desde $199 \mathrm{GPa}$ hasta $211 \mathrm{GPa}$ (revenido a $500^{\circ} \mathrm{C}$ ) y \\ para el acero C55E el módulo varía desde 202 GPa hasta 209 GPa para el acero revenido a \\ $650^{\circ} \mathrm{C}$. El presente trabajo se centra en la caracterización estructural de los tres aceros \\ mencionados a los distintos estados de tratamiento térmico, utilizando las técnicas de \\ microscopía óptica de reflexión y microscopía electrónica de barrido, y se propone una \\ explicación de la variación del módulo a partir del comportamiento de las dislocaciones y \\ su interacción con átomos de soluto y con otras dislocaciones.
}

Palabras clave Módulo de Young. Tratamiento térmico. Aceros al carbono. Ultrasonidos.

\section{Change on Young's modulus of hipoeutectoide carbon steels with heat treatment}

\begin{abstract}
In previous papers, several hipoeutectoide carbon steels in quenched condition showed values of Young's modulus inferior to values corresponding to tempered condition. In all cases, Young's modulus was measured by ultrasound techniques. In fact, modulus of C22E steel (EN 10083) increases from $209 \mathrm{GPa}$ (quenched condition) up to $211 \mathrm{GPa}$ (tempered to $650^{\circ} \mathrm{C}$ ); for $\mathrm{C} 45 \mathrm{E}$ steel, modulus increases from $199 \mathrm{GPa}$ to $211 \mathrm{GPa}$ (tempered to 500 ${ }^{\circ} \mathrm{C}$ ), and for C55E steel Young's modulus varies from $202 \mathrm{GPa}$ to $209 \mathrm{GPa}$ for tempered steel to $650^{\circ} \mathrm{C}$. The present work focuses on the microstructural characterization of these steels at different heat conditions using reflection optical microscopy and also scanning electronic microscopy and the authors propose an explanation of the modulus variation using the behaviour of the dislocations array and their interaction with solute atoms and other dislocations present in steel.
\end{abstract}

Keywords Young's modulus. Heat treatment. Carbon steels. Ultrasound.

\section{INTRODUCCIÓN}

Es bien conocido que el módulo de Young de aleaciones metálicas experimenta variaciones de entre 1 y $10 \%$ con el trabajo mecánico en frío o con los tratamientos térmicos y existen numerosos trabajos que presentan una revisión exhaustiva de dichos cambios. Entre ellos cabe mencionar la revisión efectuada por Mack ${ }^{[1]}$ y un trabajo de los autores del presente estudio ${ }^{[2]}$. No obstante, estas variaciones no parecen relevantes en cuestiones relacionadas con el diseño de componentes o estructuras porque los factores de seguridad utilizados son altos y minimizan los efectos derivados de esta variación. Por el contrario, estos cambios en el módulo de Young pueden afectar de manera significativa la bondad de la simulación numérica de procesos de conformado.

En el presente trabajo se ha efectuado una amplia caracterización estructural de los aceros C22E, C45E y C55E (EN 10083) en los estados de tratamiento de temple y revenidos a distintas temperaturas utilizando técnicas de microscopía óptica de reflexión y de microscopía electrónica de barrido. Igualmente, se propone una explicación de la variación del módulo de Young a partir del

(*) Departament de Ciència dels Materials i Enginyeria Metallúrgica, Facultat de Química, Universitat de Barcelona, Martí i Franquès 1, 08028 Barcelona.

(**) Departament de Ciència dels Materials i Enginyeria Metal.lúrgica, Universitat Politécnica de Catalunya, Comte d'Urgell 187, 08036 Barcelona. 
comportamiento de las dislocaciones referenciado por otros autores y de su interacción con átomos de soluto y con otras dislocaciones.

\section{VARIACIÓN DEL MÓDULO DE YOUNG CON EL TRATAMIENTO TÉRMICO EN LOS ACEROS C22E, C45E Y C55E}

I. Fonseca et al. ${ }^{[2]}$ determinaron la variación del módulo de Young (E) del acero C45E con el tratamiento térmico. Las medidas de E se llevaron a cabo por ensayo mecánico y también mediante técnicas de ultrasonidos. Los resultados obtenidos se muestran en la tabla I. Una primera consideración a efectuar es la gran concordancia entre los resultados obtenidos por ensayo mecánico y mediante ultrasonidos, tanto en el material templado como en los distintos revenidos y, también, en el recocido total efectuado.

Se observa un valor de módulo (E) relativamente bajo en el material templado (199-200 $\mathrm{GPa})$ y un incremento de este parámetro con el revenido hasta alcanzar un máximo a $500^{\circ} \mathrm{C}(211$. $212 \mathrm{GPa})$; y una vez alcanzado este valor, $\mathrm{E}$ decrece, de nuevo, hasta 206-207 GPa en el material recocido.

Villuendas et al..$^{[3 \text { y } 4]}$ estudiaron la variación del módulo de Young con el tratamiento térmico en los aceros C22E y C55E. Las medidas de este parámetro se llevaron a cabo mediante técnicas de ultrasonidos. Los resultados obtenidos se incluyen en la tabla II. En ambos casos, e igual que para el acero C45E, el menor valor de módulo se obtuvo en el material templado. Con el revenido, el módulo recupera y, en ambos casos, alcanza su valor máximo en el material recocido. Cabe destacar que el acero $\mathrm{C} 22 \mathrm{E}$, con el menor porcentaje de carbono, presenta una mínima variación entre el valor del material templado (209 $\mathrm{GPa}$ ) y el material revenido

Tabla I. Acero C45E: Variación del módulo de Young con el tratamiento térmico

Table I. C45E steel: Change of Young's modulus with heat treatment

\begin{tabular}{lcc}
\hline Estado de tratamiento & $\mathrm{E}_{\text {tracción }}$ (GPa) & $\mathbf{E}_{\text {ultrasonidos }}(\mathrm{GPa})$ \\
\hline temple & 199 & 200 \\
temple+revenido $350^{\circ} \mathrm{C}$ & 207 & 209 \\
temple+revenido $500^{\circ} \mathrm{C}$ & 211 & 212 \\
temple+revenido $700^{\circ} \mathrm{C}$ & 208 & 210 \\
Recocido total & 206 & 207 \\
\hline
\end{tabular}

Rev. Metal. Madrid Vol. Extr. (2005) 46-52
Tabla II. Aceros C22E y C55E: Variación de E con el tratamiento térmico

Table II. C22E and C55E steels: Change of E with heat treatment

\begin{tabular}{lcc}
\hline Estado de tratamiento & $\mathrm{E}_{\mathrm{C} 22 \mathrm{E}}(\mathrm{GPa})$ & $\mathrm{E}_{\mathrm{C} 55 \mathrm{E}}(\mathrm{GPa})$ \\
\hline temple & 209 & 202 \\
temple+revenido $150^{\circ} \mathrm{C}$ & 209 & 203 \\
temple+revenido $250^{\circ} \mathrm{C}$ & 210 & 205 \\
temple+revenido $350^{\circ} \mathrm{C}$ & 210 & 206 \\
temple+revenido $450^{\circ} \mathrm{C}$ & 211 & 208 \\
temple+revenido $550^{\circ} \mathrm{C}$ & 211 & 209 \\
temple+revenido $650^{\circ} \mathrm{C}$ & 211 & 209 \\
recocido total & 214 & 209 \\
\hline
\end{tabular}

(211 GPa), mientras que la variación es mucho más acusada en el acero C55E (202 GPa para el material templado y $209 \mathrm{GPa}$ para al material revenido a 550 y $650^{\circ} \mathrm{C}$ ).

\section{CARACTERIZACIÓN ESTRUCTURAL: ACEROS C22E, C45E Y C55E}

\subsection{Materiales}

Las muestras se obtuvieron de barra redonda de 20 $\mathrm{mm}$ de diámetro; el acero C22E se obtuvo en estado de laminación en caliente y los aceros C45E y C55E en forma de barra calibrada. Para los tratamientos térmicos se cortaron probetas de $4 \mathrm{~mm}$ de espesor (C22E) y de $8 \mathrm{~mm}$ (C45E y C55E). Las condiciones de los tratamientos térmicos aplicados fueron las habituales en estos aceros: temple en agua desde la temperatura de austenización y revenidos en hornos de mufla al aire, enfriamiento posterior en agua ${ }^{[2-4]}$.

\subsection{Caracterización de los materiales a los dis- tintos tratamientos térmicos}

Para la caracterización estructural, se eliminó la capa de óxido y la zona decarburada. Después, las muestras se pulieron y atacaron con nital al $2 \%$, tanto para la observación por microscopía óptica como para la observación por microscopía electrónica.

En las figuras 1 (a-c) se muestran, respectivamente, las microestructuras correspondientes a aceros C22E, C45E y C55E en estado de temple, y en las figuras $2(\mathrm{a}-\mathrm{c})$, se muestran imágenes electrónicas obtenidas por MEB de los mismos 
Variación del módulo de Young con el tratamiento térmico en aceros al carbono hipoeutectoides

A. VILLUENDAS, J. JORBA Y A. ROCA
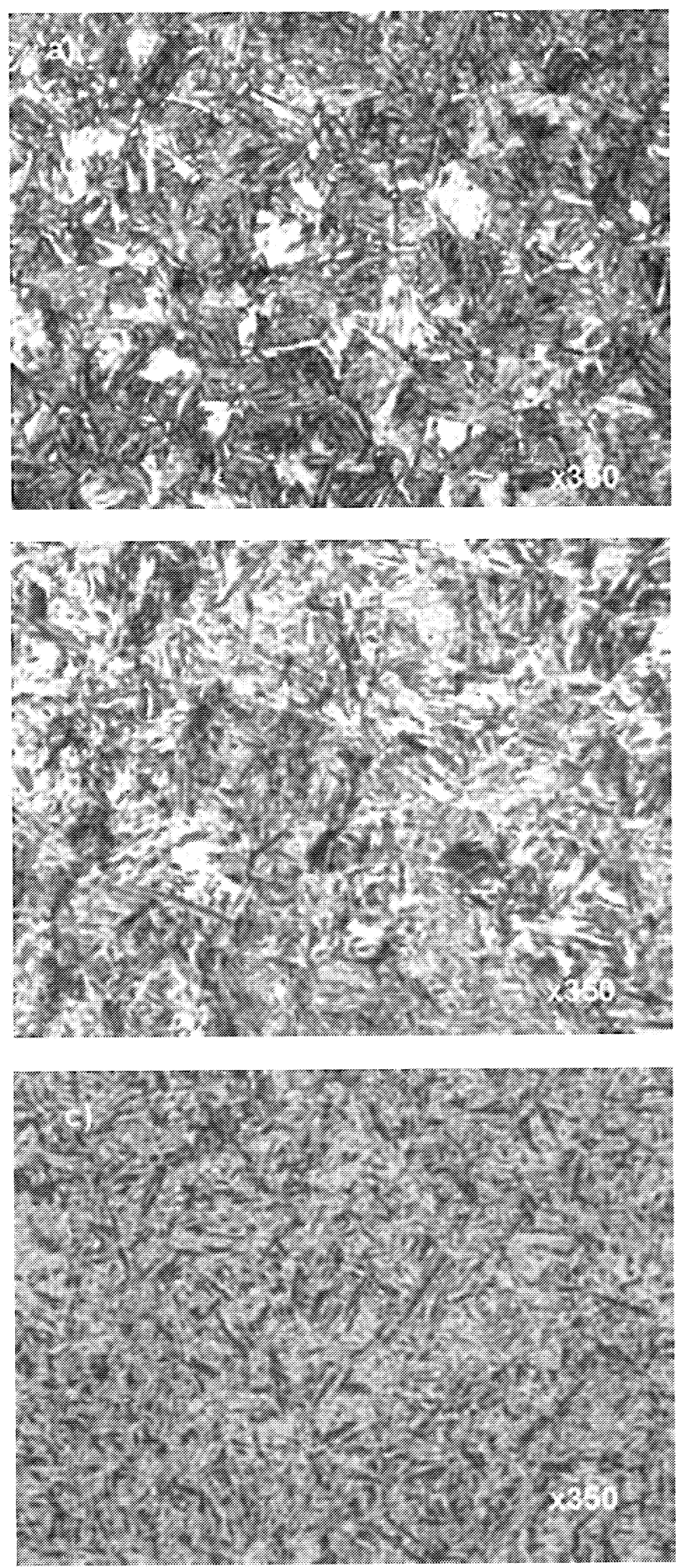

Figura 1. Micrografías ópticas de los aceros templados: a) $\mathrm{C} 22 \mathrm{E}$; b) $\mathrm{C} 45 \mathrm{E}$; c) C55E.

Figure 1. Optical micrographs of quenched steels: a) C22E; b) $C 45 E$; c) C55E.

materiales templados. La dureza obtenida después de temple fue 39, 52 y 63 HRC. Las micrografías mostradas y los valores de dureza obtenidos indican que los tratamientos térmicos fueron los adecuados, aunque puede haber un pequeño porcentaje de ferrita en el acero C22E dada la baja templabilidad de este material. También se aprecia
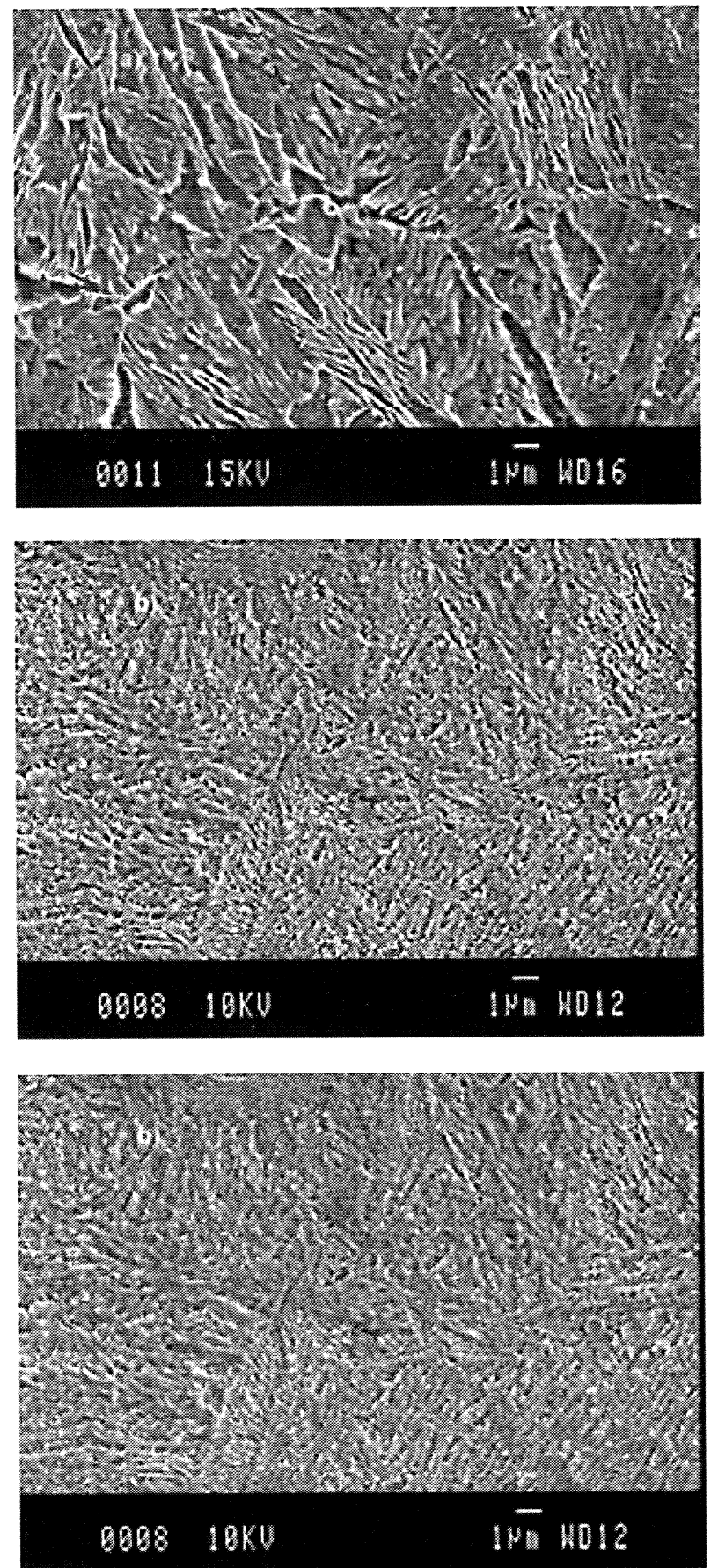

Figura 2. Micrografías $M E B$ de los aceros templados: a) $C 22 E$, b) $C 45 E$, c) C55E.

Figure 2. SEM images of quenched steels: a) C22E, b) C45E, c) C55E.

un tránsito desde una estructura de martensita acicular propia de aceros con bajos contenidos de carbono (C22E) hasta una estructura mixta formada por martensita acicular mucho más compacta y algunas zonas de martensita en placas propia de aceros de medio carbono (C55E).

En las figuras $3(\mathrm{a}-\mathrm{c})$ se muestran las microestructuras obtenidas por MEB de los mismos aceros 

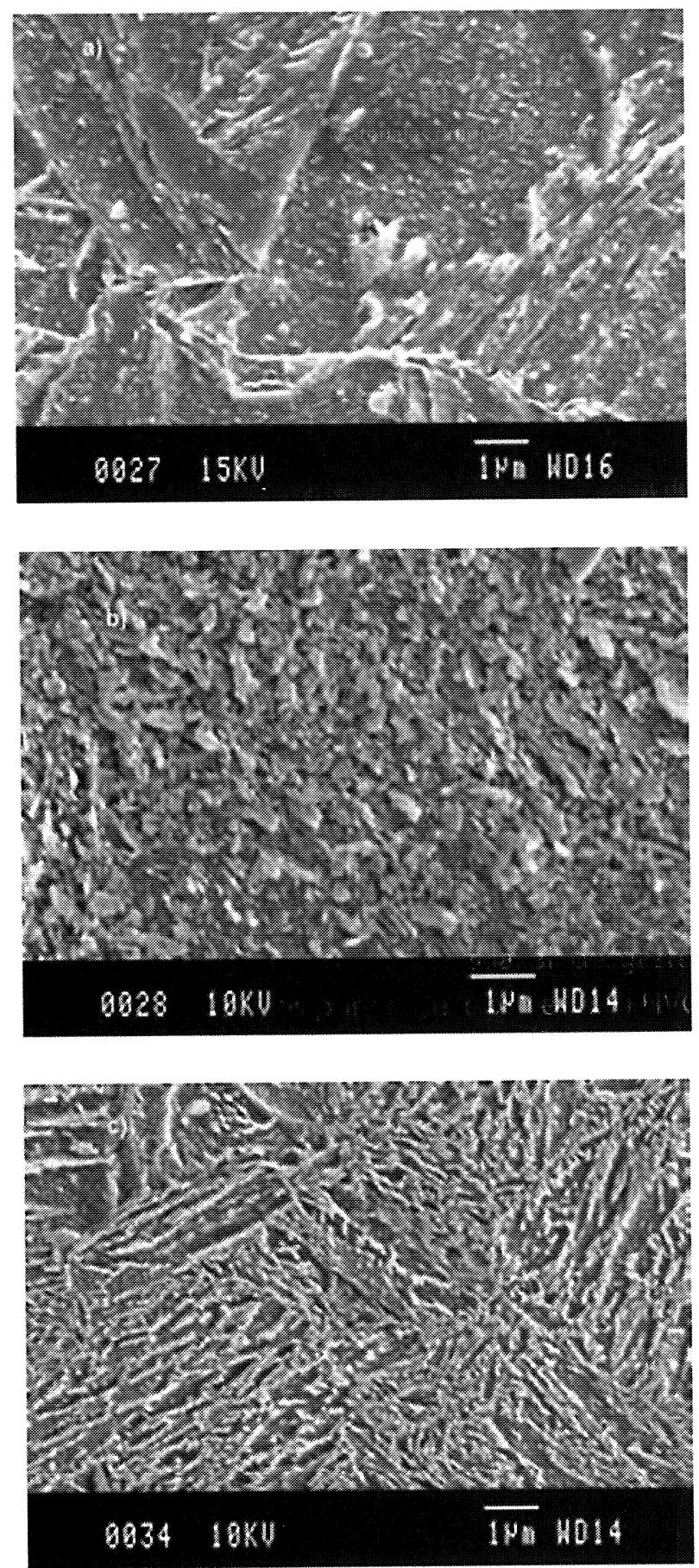

Figura 3. Micrografías $M E B$ de los aceros revenidos a $350^{\circ} \mathrm{C}$ a) $\mathrm{C} 22 \mathrm{E}$, b) $\mathrm{C} 45 \mathrm{E}$, c) C55E.

Figure 3. SEM images of quenched and tempered steels at $350^{\circ} \mathrm{C}$ : a) $C 22 \mathrm{E}$, b) $\mathrm{C} 45 \mathrm{E}$, c) $\mathrm{C} 55 \mathrm{E}$.

después del temple y revenido a $350^{\circ} \mathrm{C}$, mientras que las figuras $4(\mathrm{a}-\mathrm{c})$ corresponden a revenidos aplicados a temperaturas intermedias: C22E (450 $\left.{ }^{\circ} \mathrm{C}\right), \mathrm{C} 45 \mathrm{E}\left(500^{\circ} \mathrm{C}\right)$ y $\mathrm{C} 55 \mathrm{E}\left(450^{\circ} \mathrm{C}\right)$. Las figuras 5 (a-c), corresponden a revenidos a temperatura alta: C22E $\left(650^{\circ} \mathrm{C}\right), \mathrm{C} 45 \mathrm{E}\left(700^{\circ} \mathrm{C}\right), \mathrm{C} 55 \mathrm{E}(650$ $\left.{ }^{\circ} \mathrm{C}\right)$, mientras que las figuras $6(\mathrm{a}-\mathrm{c})$ corresponden
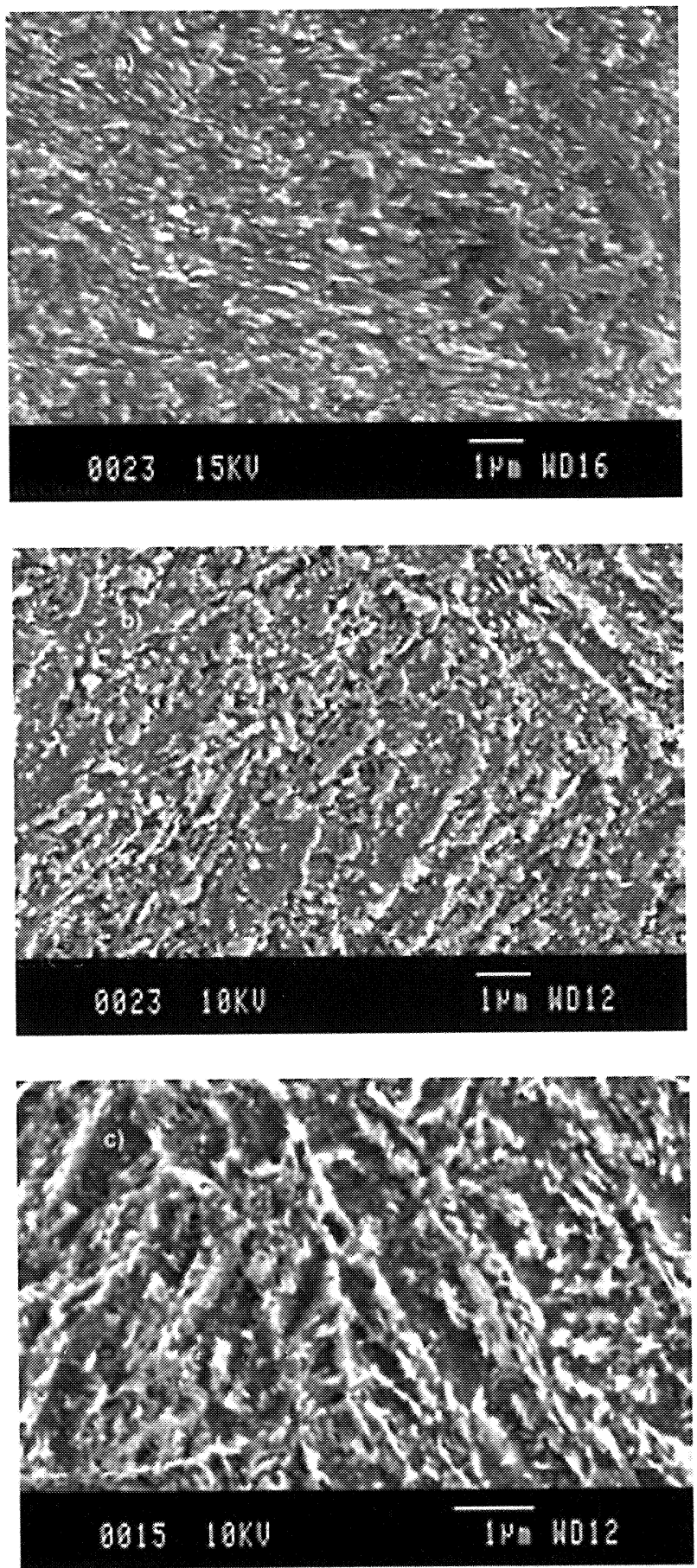

Figura 4. Micrografías $M E B$ de los aceros revenidos a temperaturas intermedias: a) $\mathrm{C} 22 \mathrm{E}\left(450^{\circ} \mathrm{C}\right)$, b) C45E (500 $\left.{ }^{\circ} \mathrm{C}\right)$, c) C55E $\left(450^{\circ} \mathrm{C}\right)$.

Figure 4. SEM images of quenched and tempered steels at: a) $\mathrm{C} 22 \mathrm{E}, 450^{\circ} \mathrm{C}$; b) $\mathrm{C} 45 \mathrm{E}, 500^{\circ} \mathrm{C}$; c) $\mathrm{C} 55 \mathrm{E}, 450^{\circ} \mathrm{C}$.

a los mismos aceros y condiciones obtenidas a mayores aumentos.

En esta secuencia de micrografías se aprecia una evolución en el tamaño y distribución de los carburos formados durante el revenido a distintas temperaturas. Así, es difícil apreciar los carburos 
Variación del módulo de Young con el tratamiento térmico en aceros al carbono hipoeutectoides
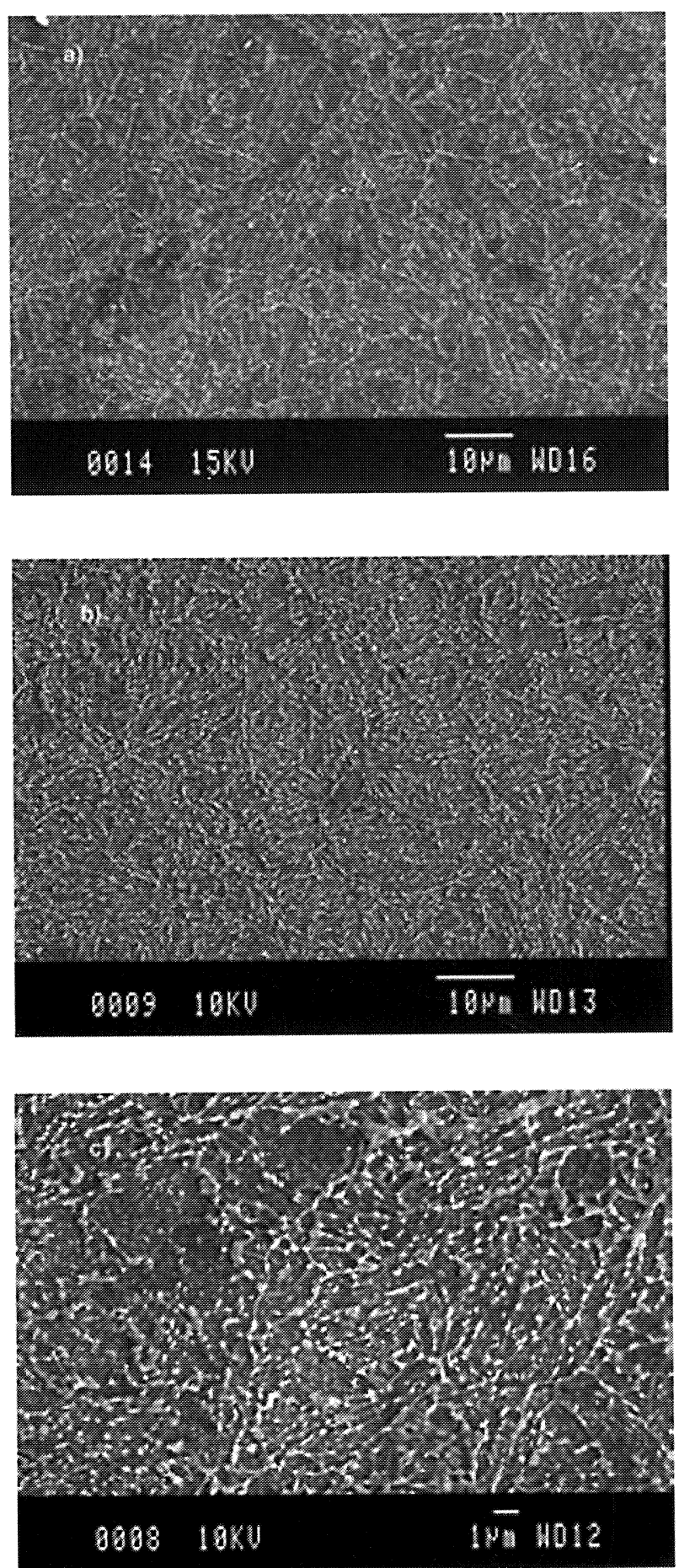

Figura 5. Micrografías $M E B$ de los aceros revenidos a temperaturas altas: a) $\mathrm{C} 22 \mathrm{E}\left(650^{\circ} \mathrm{C}\right)$, b) $\mathrm{C} 45 \mathrm{E}\left(700^{\circ} \mathrm{C}\right)$, c) $\mathrm{C} 55 \mathrm{E}\left(650^{\circ} \mathrm{C}\right)$.

Figure 5. SEM images of quenched and tempered steels at: a) $C 22 \mathrm{E}, 650^{\circ} \mathrm{C}$; b) $\mathrm{C} 45 \mathrm{E}, 700^{\circ} \mathrm{C}$; c) $\mathrm{C} 55 \mathrm{E}, 650^{\circ} \mathrm{C}$.

formados durante el revenido a $350^{\circ} \mathrm{C}$ que, en todo caso, son de pequeño tamaño y aparecen distribuidos en la matriz o perfilan los bordes de las agujas y placas de la martensita revenida (C55E). Al aumentar la temperatura de revenido aumenta el
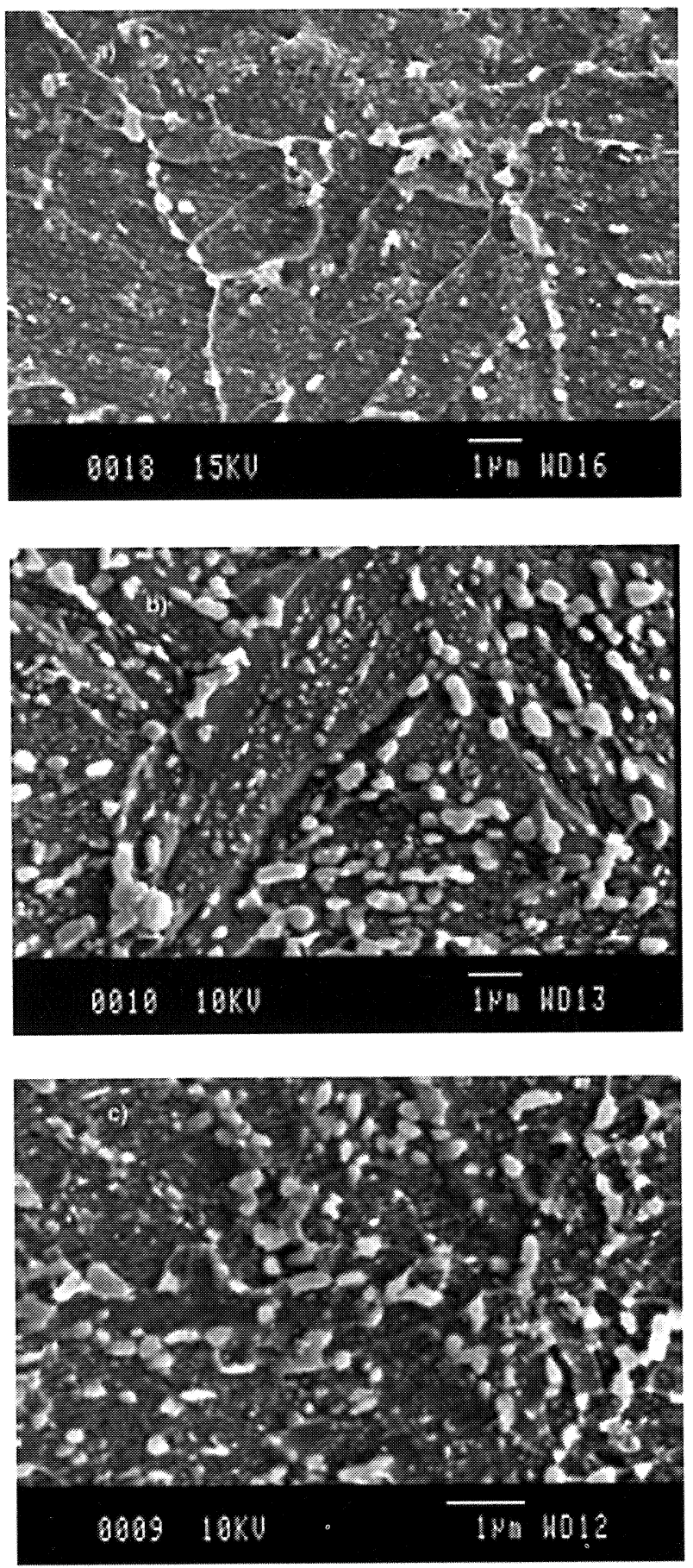

Figura 6. Micrografías $M E B$ de los aceros revenidos a temperaturas altas: a) $\mathrm{C} 22 \mathrm{E}\left(650^{\circ} \mathrm{C}\right)$, b) $\mathrm{C} 45 \mathrm{E}\left(700^{\circ} \mathrm{C}\right)$, c) $\mathrm{C} 55 \mathrm{E}\left(650^{\circ} \mathrm{C}\right)$.

Figure 6. SEM images of quenched and tempered steels at: a) $\mathrm{C} 22 \mathrm{E}, 650^{\circ} \mathrm{C}$; b) $\mathrm{C} 45 \mathrm{E}, 700^{\circ} \mathrm{C}$; c) $\mathrm{C} 55 \mathrm{E}, 650^{\circ} \mathrm{C}$.

tamaño de los carburos que van coalesciendo, hasta que a revenidos a temperaturas altas se distribuyen formando rosarios alrededor de límites de grano (C22E, C45E) o agrupaciones que liberan zonas más o menos extensas de material sin apenas carburos (C45E, C55E).

Rev. Metal. Madrid Vol. Extr. (2005) 46-52 


\subsection{Discusión de resultados}

En los tres aceros estudiados, el material templado siempre presenta el menor valor de módulo de Young. Desde este valor mínimo para cada acero, el módulo se recupera con el revenido a diferentes temperaturas (Tablas I y II). Como es bien sabido, el bajo contenido de carbono del acero C22E genera una baja distorsión de la red martensítica, como así lo confirman los relativamente bajos valores de dureza obtenidos. Por esta misma razón, el mayor contenido en carbono de los aceros C45E y C55E genera una mayor distorsión de la red cristalina y una mayor dureza del material en estado de temple. Por otra parte, el tipo de martensita acicular formada en aceros de bajo-medio carbono contiene una elevada densidad de dislocaciones que es comparable con materiales altamente deformados en frío, mientras que la martensita en placas presente en aceros de alto porcentaje en carbono no precisa de la presencia de dislocaciones para formarse.

El módulo de Young del acero C22E varía el $1 \%$ si se compara entre el material templado y el material revenido, mientras que el mayor cambio de $\mathrm{E}$ se observa en el acero $\mathrm{C} 45 \mathrm{E}$ con una variación que alcanza el $6 \%$ entre el material templado y el revenido a $500{ }^{\circ} \mathrm{C}$, con una ligera disminución de $\mathrm{E}$ para un revenido a $700{ }^{\circ} \mathrm{C}$. La variación de módulo en el acero C55E es más moderada $(3,5 \%)$.

Para explicar este comportamiento puede utilizarse el razonamiento de Mott ${ }^{[5]}$, que postula que el módulo de Young varía con la presencia de dislocaciones según la expresión:

$$
\Delta \mathrm{E} / \mathrm{E}=-\rho l^{2} / 6 \alpha
$$

donde $\rho$ es la densidad de dislocaciones del material, $l$, la longitud de las dislocaciones o de los segmentos de dislocación entre puntos de anclaje y $\alpha$, un parámetro relacionado directamente con esta longitud. Según esta expresión, a medida que aumenta $\rho$ o $l$ se produce una mayor disminución de E. Igualmente, Granato y Lücke ${ }^{[6]}$ indican que las dislocaciones del material pueden anclarse con otras dislocaciones o con defectos de punto, y según estos autores, cuando se aplica un esfuerzo subelástico sobre el material, además de la deformación elástica derivada de la elasticidad de los enlaces, existe una deformación asociada al arqueo de las dislocaciones ancladas, lo que conduce a una disminución del módulo de Young del material.
En este contexto, el acero C22E templado y revenido debe presentar una densidad de dislocaciones comparativamente baja y deben existir pocos puntos de anclaje derivados de la intersección entre dislocaciones o la presencia de átomos de soluto, mientras que el número de intersecciones debe ser mayor en los aceros con mayor porcentaje de carburos (C45E y C55E). Este aspecto puede observarse en las figuras $3-6$, en las que se observa una creciente densidad de precipitados de cementita.

Los trabajos de Mott, Granato y Lücke justifican los valores bajos del módulo en los materiales templados de los aceros de mayor contenido en carbono, debido al arqueo de las dislocaciones existentes en los mismos. En los materiales revenidos, parecen existir dos procesos contrapuestos: uno, la disminución de la densidad de dislocaciones provoca una recuperación de los valores de E respecto al material templado y, otro, que generaría una disminución de módulo por pérdida de puntos de anclaje de las dislocaciones derivado de la coalescencia de los carburos de hierro. Este segundo efecto puede observarse en las figuras 3-6. Así, el comportamiento del material C45E podría explicarse de la forma siguiente $^{[2]}$ : el material templado presenta un módulo de 199-200 GPa debido a la elevada densidad de dislocaciones del material y al elevado número de puntos de anclaje que derivan de la presencia de carbono sobresaturando la red de la martensita. El revenido a temperaturas crecientes y hasta $500{ }^{\circ} \mathrm{C}$ conduce a un aumento del módulo que podría asociarse fundamentalmente a la drástica disminución de la densidad de dislocaciones; en el revenido a $700{ }^{\circ} \mathrm{C}$ el módulo disminuye ligeramente lo que se debería al aumento de la longitud de segmentos de dislocación anclados (Figs. 3-6).

En relación con el acero C55E, los aceros de contenido superior a $0,5 \%$ presentan microestructuras con martensita formada por mezcla de dislocaciones y maclas ${ }^{[7]} y$, en consecuencia, con una menor densidad de dislocaciones. En estos aceros también existen dos mecanismos en competencia: por un lado, al aumentar el contenido de carbono debería aumentar la densidad de dislocaciones y por otro, que disminuye el porcentaje de esta martensita a partir de un determinado contenido de carbono. Ello conduciría a una suavización en el cambio del módulo de Young.

\section{Agradecimientos}

Los autores agradecen al Ministerio de Ciencia y Tecnología por la subvención concedida a través 
Variación del módulo de Young con el tratamiento térmico en aceros al carbono hipoeutectoides A. Villuendas, J. JORBA Y A. ROCA

del Plan Nacional MAT97-0827-C02-02 que ha permitido financiar una parte de este trabajo. Los autores también desean agradecer a la Sra. Esther Vilalta por su colaboración en la preparación de muestras para su observación metalográfica.

\section{REFERENCIAS}

[1] D.J. MACK, Trans. AIME (166) 1946, 68-85.

[2] I. FonseCA, J.A. Benito, I. MejíA, J. Jorba y A. Roca, Rev. Metal. Madrid 38 (2002) 249-255.
[3] A. Villuendas, Máster en Química Experimental, Universitat de Barcelona, 2002.

[4] J. Jorba, A. Roca y A. Villuendas, VIII Cong. Nac. Propiedades Mecánicas de Sólidos, Ed. V. Amigó, Gandía, 2002, pp. 497-504.

[5] N.F. MotT, Phil. Mag. 43 (1952) 1.151-1.178.

[6] H.F. Pollard, Sound Waves in Solids, Pion Ltd., London, 1977, pp. 247-260.

[7] D.A. Porter y K.E. EAsterling, Phase Transformation in Metals and Alloys, Van Nostrand Reinhold Co. Ltd., UK., 1981. 